Research Article

\title{
Sharp Bound of the Number of Zeros for a Liénard System with a Heteroclinic Loop
}

\author{
Junning Cai $\mathbb{D},{ }^{1}$ Minzhi Wei $\mathbb{D}^{1},{ }^{1}$ and Guoping Pang ${ }^{2}$ \\ ${ }^{1}$ Department of Applied Mathematics, Guangxi University of Finance and Economics, Nanning, Guangxi 530003, China \\ ${ }^{2}$ School of Mathematics and Statistics, Yulin Normal University, Yulin 537000, China \\ Correspondence should be addressed to Minzhi Wei; xiaoyanxiong123@163.com
}

Received 23 November 2020; Revised 24 December 2020; Accepted 8 January 2021; Published 28 January 2021

Academic Editor: Binxiang Dai

Copyright (c) 2021 Junning Cai et al. This is an open access article distributed under the Creative Commons Attribution License, which permits unrestricted use, distribution, and reproduction in any medium, provided the original work is properly cited.

In the presented paper, the Abelian integral $I(h)$ of a Liénard system is investigated, with a heteroclinic loop passing through a nilpotent saddle. By using a new algebraic criterion, we try to find the least upper bound of the number of limit cycles bifurcating from periodic annulus.

\section{Introduction}

A well-known analytic system with planar polynomial differential equation of degree $n$ is of the form:

$$
\dot{x}=P_{n}(x, y), \quad \dot{y}=Q_{n}(x, y) .
$$

In 1977, Arnold [1] proposed weak Hilbert's 16th problem and studied the number of zeros of the Abelian integral:

$$
I(h, \delta)=\oint_{\Gamma_{h}} q \mathrm{~d} x-p \mathrm{~d} y, \quad h \in J,
$$

where $p$ and $q$ are the polynomials of degree $n \geq 2$ and $\Gamma_{h}$ are some closed ovals of corresponding Hamiltonian. More precisely, $H(x, y)$ is the Hamiltonian function of special form of (1):

$$
\begin{aligned}
& \dot{x}=H_{y}+\varepsilon p(x, y, \delta), \\
& \dot{y}=-H_{x}+\varepsilon q(x, y, \delta),
\end{aligned}
$$

where $H(x, y), p(x, y)$, and $q(x, y)$ are the polynomials of $x$ and $y$, their degrees satisfy $\max \{\operatorname{deg} p, \operatorname{deg} q\}=n$, $\operatorname{deg}(H)=n+1$, and $\varepsilon$ is a positive and sufficiently small parameter.
More precisely, the following Liénard system of type $(m, n)$ attracted more and more attentions from mathematicians [2-15]:

$$
\begin{aligned}
& \dot{x}=y, \\
& \dot{y}=f(x)+\varepsilon g(x) y,
\end{aligned}
$$

where $f(x)$ and $g(x)$ are the polynomials of degrees $m$ and $n$, respectively. For example, Wang and Xiao [16] concluded that the number of limit cycles in the system bifurcating from period annulus is at most three. Qi and Zhao [17] considered the Liénard system of type $(5,3)$, [18], Asheghi and Zangeneh [19] considered the Liénard system of type (5, $4)$, and Sun [20] studied the limit cycles of type $(7,6)$ with a heteroclinic loop connecting two nilpotent saddles. In this paper, we intend to study on a following Liénard system that is a small perturbation of the Hamiltonian vector field:

$$
\begin{aligned}
& \dot{x}=y, \\
& \dot{y}=x(x+1)^{3}\left(x+\frac{1}{3}\right)+\varepsilon\left(a_{1} x+a_{2} x^{3}+a_{3} x^{5}+x^{7}\right) y,
\end{aligned}
$$

with $0<\varepsilon \ll 1$, and $a_{1}, a_{2}, a_{3}$ are the constants. Equation (5) holds the hyperelliptic Hamiltonian function: 
$\bar{H}(x, y)=\frac{1}{2} y^{2}-\frac{1}{6} x^{6}-\frac{2}{3} x^{5}-x^{4}-\frac{2}{3} x^{3}-\frac{1}{6} x^{2}=\frac{1}{2} y^{2}+A(x)$.

The level sets (i.e., $\bar{H}(x, y)=h$ ) of the Hamiltonian function (6) are sketched in Figure 1. $\bar{H}(x, y)=h$ defines a family of closed orbits of system (5) $\mid \varepsilon=0$, denoted by $\left\{\Gamma_{h}\right\}$. $\Gamma_{0}$ is the corresponding orbit to $h=0$, it incloses an elementary center $(-(1 / 3), 0)$, and $\Gamma_{-(8 / 2187)}$ defines two heteroclinic orbits, connecting a nilpotent saddle $(-1,0)$ and a hyperbolic saddle $(0,0)$. The Melnikov function on $\Gamma_{h}$ is

$$
\begin{aligned}
I(h, \delta) & =\oint_{\Gamma h}\left(a_{1} x+a_{2} x^{3}+a_{3} x^{5}+x^{7}\right) y \mathrm{~d} x \\
& \equiv a_{1} I_{1}(h)+a_{2} I_{2}(h)+a_{3} I_{3}(h)+I_{4}(h),
\end{aligned}
$$

for $h \in(-(8 / 2187), 0)$, where $\delta=\left(a_{1}, a_{1}, a_{3}, 1\right)$ and $I_{i}(h)=\oint_{\Gamma h} x^{2 i-1} y \mathrm{~d} x, i=1,2,3,4$. Our main work is to provide a complete description of the number of limit cycles for perturbed system in the whole plane.

\section{Some Preliminaries}

For system (3), some related definitions and significative results are introduced, it can be seen in [21-23] in detail.

Definition 1. Assume that $f_{0}, f_{1}, f_{2}, \ldots, f_{n-1}$ are analytic functions on a real open interval $J$.

(i) The family of sets $\left\{f_{0}, f_{1}, f_{2}, \ldots, f_{n-1}\right\}$ is called a Chebyshev system (T-system for short) provided that any nontrivial linear combination $k_{0} f_{0}(x)+$ $k_{1} f_{1}(x)+\cdots+k_{n-1} f_{n-1}(x)$ has at most $n-1$ isolated zeros on $J$.

(ii) An ordered set of $n$ functions $\left\{f_{0}, f_{1}, f_{2}, \ldots, f_{n-1}\right\}$ is called a complete Chebyshev system (CT-system

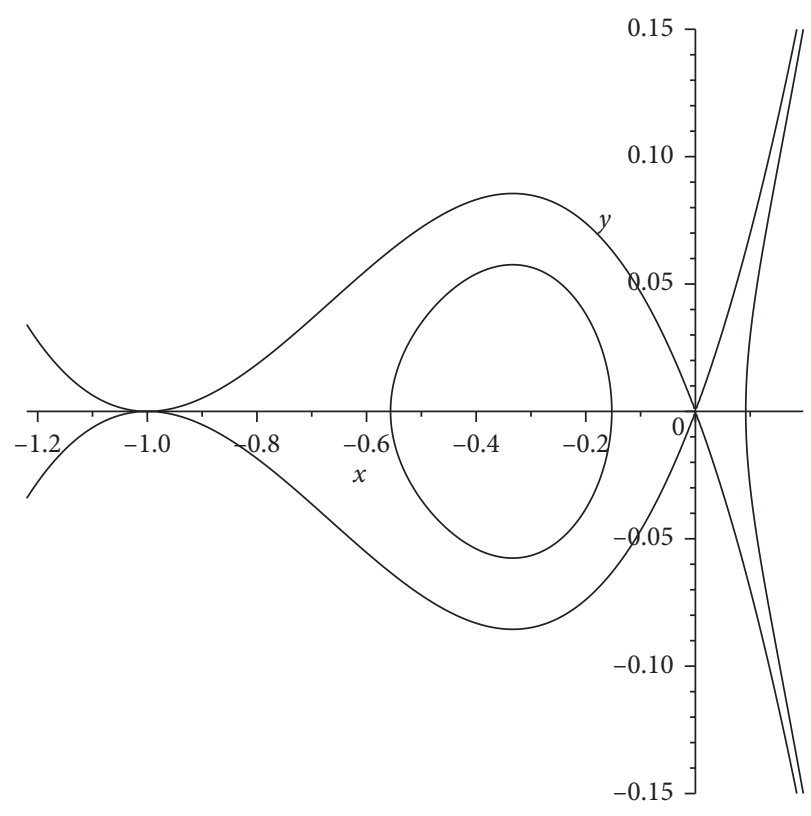

FIGURE 1: The level set of $H(x, y)$.

for short) provided any nontrivial linear combination $k_{0} f_{0}(x)+k_{1} f_{1}(x)+\cdots+k_{n-1} f_{n-1}(x)$ has at most $i-1$ zeros for all $i=1,2, \ldots, n$. Moreover, it is called an extended complete Chebyshev system (ECT-system for short) if the multiplicities of zeros are taken into account.

(iii) The continuous Wronskian of $\left\{f_{0}, f_{1}, f_{2}, \ldots, f_{n-1}\right\}$ at $x \in R$ is

$$
W\left[f_{0}, f_{1}, f_{2}, \ldots, f_{k-1}\right]=\operatorname{det}\left(f_{i}^{j}\right)_{0 \leq i, j \leq k-1}=\left|\begin{array}{cccc}
f_{0}(x) & f_{1}(x) & \ldots & f_{k-1}(x) \\
f_{0}^{\prime}(x) & f_{1}^{\prime}(x) & \ldots & f_{k-1}^{\prime}(x) \\
\vdots & \ldots & \ldots & \vdots \\
f_{0}^{(k-1)}(x) & f_{1}^{(k-1)}(x) & \ldots & f_{k-1}^{(k-1)}(x)
\end{array}\right|
$$

where $f^{\prime}(x)$ is the first-order derivative of $f(x)$ and $f_{i}^{j}(x)$ is the $j$ th order derivative of $f_{i}(x), i \geq 2$. The definitions imply that the function tuple $\left\{f_{0}, f_{1}, f_{2}, \ldots, f_{n-1}\right\}$ is an ECTsystem on $J$; therefore, it is an ECT-system on $J$ and then a T-system on $J$; however, the inverse implications are not true.

Let $H(x, y)=(1 / 2) y^{2}+A(x)$ in (5) be an analytic function. The set of ovals $\Gamma_{h}=H(x, y)=h$ inside periodic annulus is defined by $h \in\left(h_{1}, h_{2}\right)=J$. Supposed that $P$ is a punctured neighborhood of the origin foliated by ovals $\Gamma_{h}$, then the projection of $P$ on the $x$-axis is an interval $\left(x_{l}, x_{r}\right)$ with $x_{l}<0<x_{r}$. It is easy to know that $x A^{\prime}(x)>0$, $\forall x \in\left(x_{l}, x_{r}\right) \backslash\{0\}$, such that $A(x)$ has a zero of even multiplicity at $x=0$, and there exists an analytic involution $z(x)$, which is defined by $A(x)=A(z(x))$.

Lemma 1 (see [22]). On $\left(x_{r}, x_{l}\right)$, supposed that an analytic function $f_{i}(x)$ satisfies

$$
I_{i}(h)=\oint_{\Gamma_{h}} f_{i}(x) y^{2 s-1} \mathrm{~d} x, \quad \text { for } i=0,1,2, \ldots, n-1,
$$

where $h \in\left(h_{1}, h_{2}\right), s \in N$, and $I_{h}$ is the oval surrounding the origin inside the level curve $\left\{A(x)+(1 / 2) y^{2 m}=h\right\}$. Setting

$$
l_{i}(x):=\frac{f_{i}(x)}{A^{\prime}(x)}-\frac{f_{i} z((x))}{A^{\prime}(x)} .
$$


If the following assumptions are satisfied

(i) $W\left[l_{0}, l_{1}, \ldots, l_{i}\right]$ is nonvanishing on $\left(x_{l}, x_{r}\right)$ for $i=0,1, \ldots, n-2$

(ii) $W\left[l_{0}, l_{1}, \ldots, l_{n-1}\right]$ has $k$ zeros on $\left(x_{l}, x_{r}\right)$ counting with multiplicities

(iii) $s>n+k-2$

then for all nontrivial linear combination of $\left\{I_{0}, I_{1}, \ldots, I_{n-1}\right\}$ has at most $n+k-1$ zeros on $\left(h_{1}, h_{2}\right)$ counting the multiplicities. Meantime, $\left\{I_{0}, I_{1}, \ldots, I_{n-1}\right\}$ is called a T-system with accuracy $k$ on $\left(h_{1}, h_{2}\right)$, where $W\left[l_{0}, l_{1}, \ldots, l_{i}\right]$ is Wronskian of $\left\{l_{0}, l_{1}, \ldots, l_{n-1}\right\}$.

However, the third condition above always not been satisfied, so we usually apply the next lemma to increase the power of $y$ in $I_{i}$.

Lemma 2 (see [22]). Let $\Gamma_{h}$ be an oval inside the level curve $\left\{A(x)+(1 / 2) y^{2 m}=h\right\}, F(x)$ be a function which satisfies $\left(F(x) / A^{\prime}(x)\right)$ which is analytic at $x=0$. Hence,

$$
\oint_{\Gamma_{h}} F(x) y^{k-2} \mathrm{~d} x=\oint_{\Gamma_{h}} G(x) y^{k} \mathrm{~d} x, \quad \forall k \in N,
$$

where $G(x)=(1 / k)\left((F(x)) /\left(A^{\prime}(x)\right)\right)^{\prime}(x)$.

\section{The Least Upper Bound of Number of Zeros of $I(h, \delta)$}

Multiply $I_{i}(h)$ by $\left(\left(2 A(x)+y^{2}\right) / 2 h\right)=1$, and the following is obtained:

$$
\begin{aligned}
I_{i}(h) & =\frac{1}{2 h} \oint_{\Gamma h}\left(2 A(x)+y^{2}\right) x^{2 i-1} y \mathrm{~d} x \\
& =\frac{1}{2 h}\left(\oint_{\Gamma h} 2 A(x) x^{2 i-1} y \mathrm{~d} x+\oint_{\Gamma h} x^{2 i-1} y^{3} \mathrm{~d} x\right), \quad i=0,1,3 .
\end{aligned}
$$

Setting $k=3$ and $F(x)=2 x^{2 i-1} A(x)$ and quoting Lemma 2 to $\oint_{\Gamma h} 2 x^{2 i-1} A(x) y \mathrm{~d} x$ yield

$$
\oint_{\Gamma h} 2 x^{2 i-1} A(x) y \mathrm{~d} x=\oint_{\Gamma h} G_{i}(x) y^{3} \mathrm{~d} x
$$

where $\quad G_{i}(x)=(1 / 3)\left(\left(2 x^{2 i-1} A(x)\right) / A(x)\right)^{\prime}(x)=$ $\left(\left(2 x^{2 i-1}\left(3 i x^{2}+4 i x-x+i\right)\right) /\left(3(3 x+1)^{2}\right)\right)$.

By substituting (13) into (12) and multiplying $((2 A(x)+$ $\left.\left.y^{2}\right) / 2 h\right)=1$ again, it changes to

$$
\begin{aligned}
I_{i}(h)= & \frac{1}{2 h} \oint_{\Gamma h}\left(G_{i}(x)+x^{2 i-1}\right) y^{3} \mathrm{~d} x \\
= & \frac{1}{4 h^{2}} \oint_{\Gamma h}\left(2 A(x)+y^{2}\right)\left(G_{i}(x)+x^{2 i-1}\right) y^{3} \mathrm{~d} \phi \\
= & \frac{1}{4 h^{2}} \oint_{\Gamma h} 2 A(x)\left(x^{2 i-1}+G_{i}(x)\right) y^{3} \mathrm{~d} x \\
& +\frac{1}{4 h^{2}} \oint_{\Gamma h}\left(x^{2 i-1}+G_{i}(x)\right) y^{5} \mathrm{~d} x .
\end{aligned}
$$

Quoting Lemma 2, setting $k=5$ and $F(x)=2 A(x)\left(x^{2 i-1}+G_{i}(x)\right)$, the following is obtained:

$$
\oint_{\Gamma h} 2 A(x)\left(x^{2 i-1}+G_{i}(x)\right) y^{3} \mathrm{~d} x=\oint_{\Gamma h} E_{i}(x) y^{5} \mathrm{~d} x,
$$

where

$$
\begin{aligned}
E_{i}(x)= & \frac{1}{5}\left(\frac{2 A(x)\left(x^{2 i-1}+G_{i}(x)\right.}{A(x)}\right)^{\prime}(x)=\frac{2 x^{2 i-1} r_{i}(x)}{15(3 x+1)^{4}} \\
r_{i}(x)= & 81 i x^{4}+18 x^{4} i^{2}-24 x^{3}+48 i^{2} x^{3}+144 i x^{3} \\
& +44 i^{2} x^{2}+84 i x^{2}-14 x^{2}-4 x+16 i^{2} x+24 i x \\
& +3 i+2 i^{2} .
\end{aligned}
$$

By substituting (15) into (14) and multiplying $((2 A(x)+$ $\left.\left.y^{2}\right) / 2 h\right)=1$ again, the following is obtained:

$$
\begin{aligned}
I_{i}(h)= & \frac{1}{4 h^{2}} \oint_{\Gamma h}\left(E_{i}(x)+G_{i}(x)+x^{2 i-1}\right) y^{5} \mathrm{~d} x \\
= & \frac{1}{8 h^{3}} \oint_{\Gamma h}\left(2 A(x)+y^{2}\right)\left(E_{i}(x)+G_{i}(x)+x^{2 i-1}\right) y^{5} d, \\
= & \frac{1}{8 h^{3}} \oint_{\Gamma h} 2 A(x)\left(E_{i}(x)+G_{i}(x)+x^{2 i-1}\right) y^{5} \mathrm{~d} x \\
& +\frac{1}{8 h^{3}} \oint_{\Gamma h}\left(E_{i}(x)+G_{i}(x)+x^{2 i-1}\right) y^{7} \mathrm{~d} x .
\end{aligned}
$$

Quoting Lemma 2 once more, then

$\oint_{\Gamma h} 2 A(x)\left(E_{i}(x)+G_{i}(x)+x^{2 i-1}\right) y^{5} \mathrm{~d} x=\oint_{\Gamma h} D_{i}(x) y^{7} \mathrm{~d} x$,

where

$$
\begin{aligned}
D_{i}(x)= & \frac{1}{7}\left(\frac{2 A(x)\left(x^{2 i-1}+G_{i}(x)+E_{i}(x)\right.}{A(x)}\right)^{\prime}(x) \\
= & \frac{2 x^{2 i-1} \overline{g_{i}}(x)}{105(3 x+1)^{6}}, \\
\bar{g}_{i}(x)= & 15 i-24 x-1008 x^{5}-1218 x^{4}-672 x^{3}-178 x^{2} \\
& +184 i x+3645 i x^{6}+8496 i x^{5}+7761 i x^{4} \\
& +3848 i x^{3}+1107 i x^{2}+3120 i^{2} x^{3}+1072 i^{2} x^{2} \\
& +200 i^{2} x+16 i^{2}+1296 x^{6} i^{2}+4104 x^{5} i^{2} \\
& +5040 x^{4} i^{2}+544 i^{3} x^{3}+228 i^{3} x^{2}+48 i^{3} x \\
& +108 x^{6} i^{3}+432 x^{5} i^{3}+684 x^{4} i^{3}+4 i^{3} .
\end{aligned}
$$


From the above computation, the following result can be obtained easily.

\section{Lemma 3}

$$
8 h^{3} I_{i}(h)=\oint_{\Gamma h} f_{i}(x) y^{7} \mathrm{~d} x \equiv \widetilde{I}_{i}(h)
$$

where $f_{i}(x)=x^{2 i-1}+G_{i}(x)+E_{i}(x)+D_{i}(x)$. Therefore, $\left\{I_{1}(h), I_{2}(h), I_{3}(h), I_{4}(h)\right\}$ is an ECT-system if and only if $\left\{\widetilde{I}_{1}(h) t, n \tilde{I}_{2} q(h) h, \widetilde{I_{3} x}(h) 7, C \tilde{I}_{4} ;(h)\right\}$ is as well.

Take the following function

$$
l_{i}(x)=\left(\frac{f_{i}}{A^{\prime}}\right)(x)-\left(\frac{f_{i}}{A^{\prime}}\right)(z(x))
$$

where $z(x)$ is an analytic involution, defined by $A(x)=A(z(x))$ on $(-1,-(1 / 3))$. Factoring $A(x)-A(z(x))$ yields

$$
-\frac{1}{6}(x-z) q(x, z)
$$

where

$$
\begin{aligned}
q(x, z)= & z+6 z^{3}+4 z^{2}+4 z^{2} x^{2}+6 z x^{2}+x+6 z^{2} x \\
& +4 x z^{3}+x^{5}+4 x^{4}+6 x^{3}+4 x^{2}+4 x z+4 z x^{3} \\
& +4 z^{4}+x^{2} z^{3}+x^{4} z+x z^{4}+z^{2} x^{3}+z^{5}
\end{aligned}
$$

which defined $z(x)$ on $(-1,0)$. Hence,

$$
\frac{\mathrm{d}}{\mathrm{d} x} l_{i}(x)=\frac{\mathrm{d}}{\mathrm{d} x}\left(\frac{f_{i}}{A^{\prime}}\right)(x)-\left[\frac{\mathrm{d}}{\mathrm{d} x}\left(\frac{f_{i}}{A^{\prime}}\right)(z(x))\right] \frac{\mathrm{d} z}{\mathrm{~d} x},
$$

with $(d z / d x)=-(((\partial q(x, z)) / \partial x) /((\partial q(x, z)) / \partial z))$. Suppose that $x \in(-1,-(1 / 3))$, then $z(x) \in(-(1 / 3), 0)$; in other words,

$$
-1<x<-\frac{1}{3}<z<0 .
$$

Lemma 4. The function tuple $\left\{l_{1}(x), l_{2}(x), l_{3}(x), l_{4}(x)\right\}$ is an ECT-system for $x \in(-1,-(1 / 3))$.

Proof. Taking (24) into consideration, with the aid of Maple 16, we can obtain the 4 following Wronskians:

$$
\begin{aligned}
& W\left[l_{1}(x)\right]=l_{1}(x)=\frac{3(x-z) w_{1}(x, z)}{35(3 x+1)^{7}(x+1)^{3}(3 z+1)^{7}(z+1)^{3}}, \\
& W\left[l_{1}(x), l_{2}(x)\right]=\frac{18(x-z)^{3} w_{2}(x, z)}{125(z+1)^{6}(3 z+1)^{13}(x+1)^{6}(3 x+1)^{13} p(x, z)}, \\
& W\left[l_{1}(x), l_{2}(x), l_{3}(x)\right]=-\frac{108(x-z)^{6} w_{3}(x, z)}{42875(z+1)^{9}(3 z+1)^{15} z^{4}(x+1)^{9}(3 x+1)^{15} x^{4} p^{3}(x, z)}, \\
& W\left[l_{1}(x), l_{2}(x), l_{3}(x), l_{4}(x)\right]=-\frac{62208(x-z)^{10} w_{4}(x, z)}{300125(z+1)^{12}(3 z+1)^{22} z^{5}(x+1)^{12}(3 x+1)^{22} x^{5} p^{6}(x, z)}, \\
& R(q, p, z)=16 x^{6}(3 x+4)\left(27 x^{3}+54 x^{2}+27 x-4\right)(x+1)^{10} .
\end{aligned}
$$

where

$$
\begin{aligned}
p(x, z)= & x^{4}+4 x^{3}+2 x^{3} z+6 x^{2}+8 x^{2} z+3 x^{2} z^{2}+4 x \\
& +12 x z+12 x z^{2}+4 x z^{3}+1+8 z+18 z^{2}+16 z^{3} \\
& +5 z^{4}
\end{aligned}
$$

and $w_{1}(x, z), w_{2}(x, z), w_{3}(x, z)$, and $w_{4}(x, z)$ are polynomials in $\{x, z\}$ of degrees $15,32,53$, and 73 , respectively. In the following, calculating the resultant with respect to $z$ between $q(x, z)$ and $p(x, z)$ gives

From Sturm's Theorem, we know that $R(q, p, z)$ has no root on $(-1,-(1 / 3))$, then $p(x, z)$ and $q(x, z)$ have no common root on $(-1,-(1 / 3))$. In the following, we will check whether $w_{i}(x, z)$ and $q(x, z)$ have common root under the condition (25).

(i) Calculating the resultant with respect to $z$ between $q(x, z)$ and $w_{1}(x, z)$, that is, eliminating from $q(x, z)=0 \quad$ and $\quad w_{1}(x, z)=0 \quad$ gives $R\left(q, w_{1}, z\right)=15552(x+1)^{6}(3 x+1)^{6} \varphi_{1}(x)$, where 
$\varphi_{1}(x)$ is a polynomial of degree 62 in $x$. Applying Sturm's theorem to $\varphi_{1}(x)$, there is not any $x$ such that $\varphi_{1}(x)=0$, so we conclude that $W_{1}\left[l_{1}(x)\right] \neq 0$ on $(-1,-(1 / 3))$.

(ii) Calculating the resultant with respect to $z$ between $q(x, z)$ and $w_{2}(x, z)$, that is, eliminating from $q(x, z)=0$ and $w_{2}(x, z)=0$ gives $R\left(q, w_{2}, z\right)=$ $123834728448 x^{4}(3 x+1)^{10}(x+1)^{22} \varphi_{2}(x)$, where $\varphi_{2}(x)$ is a polynomial of degree 122 in $x$. Applying Sturm's theorem to $\varphi_{2}(x)$, there are three points, denoted by $x_{1}, x_{2}$, and $x_{3}$, such that $\varphi_{2}(x)=0$, which $x_{1} \approx-0.5231817697, x_{2} \approx-0.5050882970$, and $x_{3} \approx-0.39862246670$.

(iii) Thus we will check if $q(x)$ and $w_{2}(x, z)$ have any common roots on $(-1,-(1 / 3))$ by using the program with Maple 16 to find all the possible intervals.

$>$ with (RegularChains);

$>$ with (ChainTools);

$>$ with (SemiAlgebraicSetTools);

>sys: $=\left[w_{2}(x, z), q(x, z)\right]$;

$>R 2:=$ PolynomialRing $([x, z])$;

$>$ dec: $=$ Triangularize $($ sys, $R)$;

[regular_chain] $>L$ : $=\operatorname{map}($ Equations, $\operatorname{dec}, R)$; $[[x+1, z],[x, z+1],[x+1, z+1],[3 x+1,3 z+1]$,

$$
\left.\left[\varphi_{1}^{*}(x, z), \varphi_{2}^{*}(x, z)\right],\left[\eta_{1}^{*}(x, z), \eta_{2}^{*}(x, z)\right]\right],
$$

where $\varphi_{1}^{*}(x, z)=\varphi_{11}^{*}(z) x+\varphi_{12}^{*}(z), \quad \eta_{1}^{*}(x, z)=$ $\eta_{11}^{*}(z) x+\eta_{12}^{*}(z)$, and $\varphi_{11}^{*}, \varphi_{12}^{*}$, and $\eta_{2}^{*}$ are polynomials in $z$ of degree 41,41 , and $42, \eta_{11}^{*}, \eta_{12}^{*}$, and $\eta_{2}^{*}$ are polynomials in $z$ of degree 79,79 , and 80 , respectively. It is obvious that, all the roots of the regular chains $[x+1, z],[x, z+1],[x+1, z+1]$, and $[3 x+1,3 z+1]$ do not satisfy (14), the regular chains $\left[\varphi_{1}^{*}(x, z), \varphi_{2}^{*}(x, z)\right]$ and $\left[\eta_{1}^{*}(x, z), \eta_{2}^{*}(z)\right]$ are square-free and zero-dimensional (because the number of variables equals the number of polynomials). $L$ [5] [1] and $L[5][2]$ represent $\varphi_{1}^{*}(x, z)$ and $\varphi_{2}^{*}(z)$, and $L[6][1]$ and $L[6][2]$ represent $\eta_{1}^{*}(x, z)$ and $\eta_{2}^{*}(z)$ in Maple, we use the following program to check their common roots.

$>C:=\operatorname{Chain}([L[5][2], L[5][1]], \operatorname{Empty}(R 2), R 2)$; [regular_chain] $>R L$ : $=$ RealRootIsolate $\left(C, R 2\right.$, /abserr $\left.I=\left(1 / 10^{5}\right)\right)$;

[box, box] > map (BoxValues, $R L, R 2$ );

$$
\begin{aligned}
\begin{aligned}
{[x=} & {\left[-\frac{68575}{131072},-\frac{34287}{65536}\right], z=\left[-\frac{59409777128373836560319652339976029315}{340282366920938463463374607431768211456},\right.} \\
- & \left.\left.\frac{118819554256747673120639304679952058629}{680564733841876926926749214863536422912}\right]\right], \\
{[x=} & {\left[-\frac{174237}{131072},-\frac{43559}{32768}\right], z=\left[-\frac{163983771529936100306969912196931267809936064008805527989890579}{411376139330301510538742295639337626245683966408394965837152256},\right.} \\
& \left.\left.-\frac{655935086119744401227879648787725071239744256035222111959562315}{1645504557321206042154969182557350504982735865633579863348609024}\right]\right], \\
{[x=} & {\left[-\frac{5721}{32768},-\frac{22883}{131072}\right], z=\left[-\frac{95578876636133688791068498973648060853858107987}{182687704666362864775460604089535377456991567872},\right.} \\
& \left.\left.-\frac{382315506544534755164273995894592243415432431947}{730750818665451459101842416358141509827966271488}\right]\right], \\
x= & {\left[-\frac{52249}{131072},-\frac{6531}{16384}\right], } \\
z= & {\left[-\frac{3451103638335089536925233311376009}{2596148429267413814265248164610048},-\frac{6902207276670179073850466622752017}{5192296858534827628530496329220096}\right], }
\end{aligned},
\end{aligned}
$$


$>C$ : = Chain $([L[6][2], L[6][1]], \operatorname{Empty}(R), R 2)$;

[box, box] > map (BoxValues, RL, R);

[regular_chain] $>$ RL: = RealRootIsolate

$\left(C, R,{ }^{\prime}\right.$ abserr $\left.\prime=\left(1 / 10^{5}\right)\right)$;

$$
\begin{aligned}
{[[x=} & {\left[\frac{13355}{131072}, \frac{3339}{32768}\right], z=-\frac{6047230960549727002402305215747932185702389013319747}{11972621413014756705924586149611790497021399392059392}, } \\
& \left.\left.-\frac{24188923842198908009609220862991728742809556053278987}{47890485652059026823698344598447161988085597568237568},\right]\right] \\
{[x=} & {\left.\left[-\frac{66203}{131072},-\frac{33101}{65536}\right], z=\left[\frac{7002027595}{68719476736}, \frac{14004055191}{137438953472}\right]\right] }
\end{aligned}
$$

It means that there are 6 pairs of common roots of $w_{2}(x, z)$ and $q(x, z)$ in the listed intervals, respectively. However, there is not any pair of them satisfies the condition (25). It is said that $w_{2}(x, z) \neq 0 \quad$ as $\quad-1<x<-(1 / 3)$; therefore, $W\left[l_{1}(x), l_{2}(x)\right] \neq 0$.

(iii) Similarly, we use the same program as (i) and (ii) to find all the possible intervals, which may hold the common roots of $w_{3}(x, z)$ and $q(x, z)$ and then obtain the following regular chains:

$$
\begin{gathered}
{[[x, z],[x, z+1],[x+1, z],[x+1, z+1],} \\
{[3 x+1,3 z+1],\left[u_{1}^{*}(x, z), u_{2}^{*}(x, z)\right],} \\
\left.\left[\rho_{1}^{*}(x, z), \rho_{2}^{*}(x, z)\right]\right]
\end{gathered}
$$

where $\quad u_{1}^{*}(x, z)=u_{11}^{*}(z) x+u_{12}^{*}(z)$, $\rho_{1}^{*}(x, z)=\rho_{11}^{*}(z) x+\rho_{12}^{*}(z)$, and $u_{11}^{*}, u_{12}^{*}$, and $u_{2}^{*}$ are polynomials in $z$ of degree 57,57 , and 58 , respectively, $\rho_{11}^{*}, \rho_{12}^{*}(z)$, and $\rho_{2}^{*}$ are polynomials in $z$ of degree 111,111 , and 112 , respectively. Isolating the fifth and sixth regular chains yields

$$
\begin{aligned}
{[x=} & {\left[-\frac{54979}{131072},-\frac{27489}{65536}\right], z=\left[-\frac{856271130202849555762339288322376111382316862455404994678519855401}{3369993333393829974333376885877453834204643052817571560137951281152},\right.} \\
& \left.\left.-\frac{107033891275356194470292411040297013922789607806925624334814981925}{421249166674228746791672110734681729275580381602196445017243910144}\right]\right], \\
{[x=} & {\left[-\frac{174741}{131072},-\frac{43685}{32768}\right], }
\end{aligned}
$$$$
z=\left[-\frac{3030298039091615678970663582957756689217473784749385379759227090702937762986412587479826606535809219}{8749002899132047697490008908470485461412677723572849745703082425639811996797503692894052708092215296},\right.
$$$$
6060596078183231357941327165915513378434947569498770759518454181405875525972825174959653213071618437
$$$$
\left.-\frac{17498005798264095394980017816940970922825355447145699491406164851279623993595007385788105416184430592}{]}\right] \text {, }
$$$$
x=\left[-\frac{4163}{16384},-\frac{33303}{131072}\right], z=-\frac{361868533714530451234631234914240547837472188608858683748897796324263}{862718293348820473429344482784628181556388621521298319395315527974912},
$$$$
1447474134858121804938524939656962191349888754435434734995591185297051
$$$$
-\frac{3450873173395281893717377931138512726225554486085193277581262111899648}{,}
$$$$
x=\left[-\frac{42589}{32768},-\frac{170355}{131072}\right], z=-\frac{480531930410139348156667048862506606461576771541323121412001025602246951271}{904625697166532776746648320380374280103671755200316906558262375061821325312},
$$$$
961063860820278696313334097725013212923153543082646242824002051204493902541
$$

$1809251394333065553493296640760748560207343510400633813116524750123642650624]$




$$
\begin{aligned}
& x=\left[-\frac{69625}{131072},-\frac{8703}{16384}\right], z=\left[-\frac{29680061337195580870771064771100920742487699647}{22835963083295358096932575511191922182123945984},\right. \\
& \left.\left.-\frac{14840030668597790435385532385550460371243849823}{11417981541647679048466287755595961091061972992}\right]\right] \text {, } \\
& x=\left[-\frac{22699}{65536},-\frac{45397}{131072}\right], z=\left[-\frac{249398274372099944349741313215654480864719460648955}{187072209578355573530071658587684226515959365500928},\right. \\
& \left.\left.\left.\frac{124699137186049972174870656607827240432359730324477}{93536104789177786765035829293842113257979682750464}\right]\right]\right] \\
& {\left[x=\left[\frac{15487}{131072}, \frac{121}{1024}\right]\right. \text {, }} \\
& z=\left[-\frac{671186327417864448164206670006314426784341428019480859893071190393381893648895245}{1897137590064188545819787018382342682267975428761855001222473056385648716020711424},\right. \\
& \left.\left.-\frac{167796581854466112041051667501578606696085357004870214973267797598345473412223811}{474284397516047136454946754595585670566993857190463750305618264096412179005177856}\right]\right] \text {, } \\
& \left.\left[x=\left[-\frac{11593}{32768},-\frac{46371}{131072}\right], z=\left[\frac{272465990628347833}{2305843009213693952}, \frac{4359455850053565329}{36893488147419103232}\right]\right]\right] \text {. }
\end{aligned}
$$

It means that there are 8 pairs of common roots of $w_{3}(x, z)$ and $q(x, z)$ in the listed intervals, respectively. However, there is not any pair of them satisfies the condition (25). It is said that $w_{3}(x, z) \neq 0 \quad$ as $\quad-1<x<-(1 / 3)$; therefore, $W\left[l_{1}(x), l_{2}(x), l_{3}(x)\right] \neq 0$.

(iv) Similarly, we use the same program as (ii) and (iii) to find all the possible intervals, which may hold the common roots of $w_{4}(x, z)$ and $q(x, z)$ and then obtain the following regular chains:

$$
\begin{gathered}
{[[x, z],[x+1, z],[x, z+1],[x+1, z+1],} \\
{[3 x+1,3 z+1],\left[v_{1}^{*}(x, z), v_{2}^{*}(x, z)\right],} \\
\left.\left[\omega_{1}^{*}(x, z), \omega_{2}^{*}(x, z)\right]\right],
\end{gathered}
$$

where $\quad v_{1}^{*}(x, z)=\quad v_{11}^{*}(z) x+v_{12}^{*}(z), \omega_{1}^{*}$ $(x, z)=\omega_{11}^{*}(z) x+\omega_{12}^{*}(z)$, and $v_{11}^{*}, v_{12}^{*}$, and $v_{2}^{*}$ are polynomials in $z$ of degree 75,75 , and 76 , respectively, $\omega_{11}^{*}, \omega_{12}^{*}$, and $\omega_{2}^{*}$ are polynomials in $z$ of degree 145,145 , and 146, respectively. Isolating the fifth and sixth regular chains yields

$>C:=$ Chain $([L[6][2], L[6][1]], \operatorname{Empty}(R), R 2)$; [regular_chain] $>$ RL: =

RealRootIsolate $\left(C, R,{ }^{\prime}\right.$ abserr $\left.\prime=\left(1 / 10^{5}\right)\right)$;

[box, box, box, box, box, box,

box, box, box, box, box, box, box, box] > map

(BoxValues, RL, $R$ );

$>\operatorname{evalf}(\%)$;

$$
\begin{aligned}
& {[[x=[-1.113883972,-1.113876343], z=[-0.01488446846,-0.01488446846]],} \\
& {[x=[-1.277610779,-1.277603149], z=[-0.1301213260,-0.1301213260]],} \\
& {[x=[-0.5849761963,-0.5849685669], z=[-0.1345142627,-0.1345142627]],} \\
& {[x=[-1.329246521,-1.329238892], z=[-0.2715335562,-0.2715335562]],} \\
& {[x=[-0.3662719727,-0.3662643433], z=[-0.3014530812,-0.3014530812]],} \\
& {[x=[-1.333335876,-1.333328247], z=[-0.3333242893,-0.3333242893]],} \\
& {[x=[-0.3014602661,-0.3014526367], z=[-0.3662645453,-0.3662645453]],} \\
& {[x=[-0.1345214844,-0.1345138550], z=[-0.5849732646,-0.5849732646]],} \\
& {[x=[-1.078308105,-1.078300476], z=[-0.9149922188,-0.9149922188]],} \\
& {[x=[-0.9149932861,-0.9149856567], z=[-1.078306231,-1.078306231]],} \\
& {[x=[-0.01488494873,-0.01487731934], z=[-1.113876799,-1.113876799]],} \\
& {[x=[-0.1301269531,-0.1301193237], z=[-1.277609231,-1.277609231]],} \\
& {[x=[-0.2715377808,-0.2715301514], z=[-1.329244729,-1.329244729]],} \\
& [x=[-0.3333282471,-0.3333206177], z=[-1.333333333,-1.333333333]]],
\end{aligned}
$$


$>C:=$ Chain $([L[7][2], L[7][1]], \operatorname{Empty}(R), R 2)$

[regular_chain] $>$ RL: $=\operatorname{RealRootIsolate}(C$,

$R,^{\prime}$ abserr $\left.^{\prime}=\left(1 / 10^{5}\right)\right)$;

[box, box, box, box, box, box] > map (BoxValues,

$\mathrm{RL}, R)$; $>\operatorname{evalf}(\%)$;

$$
\begin{aligned}
& {[x=[0.1184310913,0.1184387207], z=[-0.3332116462,-0.3332116462]],} \\
& {[x=[0.1063232422,0.1063308716], z=[-0.4784850961,-0.4784850961]],} \\
& {[x=[0.5298614502,0.5299377441], z=[-0.7129253486,-0.7129253486]],} \\
& {[x=[-0.3332138062,-0.3332061768], z=[0.1184337894,0.1184337894]],} \\
& {[x=[-0.4784851074,-0.4784774780], z=[0.1063252093,0.1063252093]],} \\
& {[x=[-0.7129287720,-0.7129211426], z=[0.5298903953,0.5298903953]] .}
\end{aligned}
$$

It means that there are 20 pairs of common roots of $w_{4}(x, z)$ and $q(x, z)$ in the listed intervals, respectively. However, there is not any pair of them satisfies the condition (25). It is said that $w_{4}(x, z) \neq 0$ as $-1<x<-(1 / 3)$; therefore, $W\left[l_{1}(x), l_{2}(x), l_{3}(x), l_{4}(x)\right] \neq 0$.

Based on Lemma 1 and Lemma 4 , we obtain the following proposition:

Proposition 1. $\left\{\widetilde{I_{1}}(h) t, n \widetilde{I_{2}} q(h) h, \widetilde{I_{3} x}(h) 7, C \widetilde{I_{4}} ;(h)\right\}$ is an ECT-system and $\left\{I_{1}(h), I_{2}(h), I_{3}(h), I_{4}(h)\right\}$ is as well. Therefore there are at most 3 zeros for $I(h, \delta)$ on $h \in(-(8 / 2187), 0)$.

\section{Conclusion}

In, this work, we study the Poincare bifurcation of the Lie nard system with the form (5), and we prove 4 is the least upper bound of the number of limit cycles by the Poincare bifurcation.

\section{Data Availability}

The data used to support the findings of this study are available from the corresponding author upon request.

\section{Conflicts of Interest}

The authors declare that they have no conflicts of interest.

\section{Acknowledgments}

This work was supported by the National Natural Science Foundation of China (71862003), National Natural Science Foundation of Guangxi (2018JJA110058), Middle-Aged and Young Teachers' Basic Ability Promotion Project of Guangxi (2020KY16019 and 2020KY16020), and Guangxi University of Finance and Economics Project (2019QNA03).

\section{References}

[1] V. I. Arnold, "Arnolds problems," in Fazis, Moscow 2000Springer-Verlag, Berlin, Germany, 2004.
[2] Y. Zhao, "On the number of zeros of Abelian integrals for a polynomial Hamiltonian irregular at infinity," Journal of Differential Equations, vol. 209, no. 2, pp. 329-364, 2005.

[3] J. Li, "Hilbert's 16th problem and bifurcations of planar polynomial vector fields," International Journal of Bifurcation and Chaos, vol. 13, no. 1, pp. 47-106, 2003.

[4] M. Han and P. Yu, "Twelve limit cycles in a cubic case of the 16th Hilbert's problem," International Journal of Bifurcation and Chaos, vol. 15, no. 7, pp. 2191-2205, 2005.

[5] S. Wang and P. Yu, "Existence of 121 limit cycles in a perturbed planar polynomial Hamiltonian vector field of degree 11," Chaos, Solitons \& Fractals, vol. 30, no. 3, pp. 606-621, 2006.

[6] E. Horozov and I. Iliev, "On the number of limit cycles in perturbaations of quadratic Hamiltonian systems," Proceedings of the London Mathematical Society, vol. 69, pp. 98-108, 1994.

[7] F. Dumortier and C. Li, "Perturbations from an elliptic Hamiltonian of degree four," Journal of Differential Equations, vol. 176, no. 1, pp. 114-157, 2001.

[8] F. Dumortier and C. Li, "Perturbations from an elliptic Hamiltonian of degree four," Journal of Differential Equations, vol. 175, no. 2, pp. 209-243, 2001.

[9] F. Dumortier and C. Li, "Perturbation from an elliptic Hamiltonian of degree four-III global centre," Journal of Differential Equations, vol. 188, no. 2, pp. 473-511, 2003.

[10] F. Dumortier and C. Li, "Perturbation from an elliptic Hamiltonian of degree four-IV figure eight-loop," Journal of Differential Equations, vol. 188, no. 2, pp. 512-554, 2003.

[11] D. Xiao, "Bifurcations on a five-parameter family of planar vector field," Journal of Dynamics and Differential Equations, vol. 20, no. 4, pp. 961-980, 2008.

[12] C. Li and J. Llibre, "Uniqueness of limit cycles for Liénard differential equations of degree four," Journal of Differential Equations, vol. 252, no. 4, pp. 3142-3162, 2012.

[13] X. Sun, J. Su, and M. Han, "On the number of zeros of Abelian integral for some Liénard system of type $(4,3)$," Chaos, Solitons \& Fractals, vol. 51, pp. 1-12, 2013.

[14] X. Sun and P. Yu, "Cyclicity of periodic annulus and Hopf cyclicity in perturbing a hyper-elliptic Hamiltonian system with a degenerate heteroclinic loop," Journal of Differential Equations, vol. 269, no. 11, pp. 9224-9253, 2020.

[15] X. Sun, W. Huang, and J. Cai, "Coexistence of the solitary and periodic waves in convecting shallow water fluid," Nonlinear 
Analysis: Real World Applications, vol. 53, Article ID 103067, 2020.

[16] J. Wang and D. Xiao, "On the number of limit cycles in small perturbations of a class of hyper-elliptic Hamiltonian systems with one nilpotent saddle," Journal of Differential Equations, vol. 250, no. 4, pp. 2227-2243, 2011.

[17] M. Qi and L. Zhao, "Bifurcations of limit cycles from a quintic Hamiltonian system with a figure double-fish," International Journal of Bifurcation and Chaos, vol. 23, no. 7, Article ID 1350116, 2013.

[18] R. Asheghi and H. R. Z. Zangeneh, "Bifurcations of limit cycles from quintic Hamiltonian systems with an eye-figure loop," Nonlinear Analysis: Theory, Methods \& Applications, vol. 68, no. 10, pp. 2957-2976, 2008.

[19] R. Asheghi and H. R. Z. Zangeneh, "Bifurcations of limit cycles from quintic Hamiltonian systems with an eye-figure loop (II)," Nonlinear Analysis: Theory, Methods \& Applications, vol. 69, no. 11, pp. 4143-4162, 2008.

[20] X. Sun, "Bifurcation of limit cycles from a Liénard system with a heteroclinic loop connecting two nilpotent saddles," Nonlinear Dynamics, vol. 73, no. 1-2, pp. 869-880, 2013.

[21] M. Maza, "On triangular decompositions of algebraic varieties," in Proceedings of the MEGA-2000 Conference, Bath, UK, 2000.

[22] M. Grau, F. Mañosas, and J. Villadelprat, "A Chebyshev criterion for Abelian integrals," Transactions of the American Mathematical Society, vol. 363, no. 1, p. 109, 2011.

[23] F. Mañosas and J. Villadelprat, "Bounding the number of zeros of certain Abelian integrals," Journal of Differential Equations, vol. 251, pp. 1656-1669, 2011. 